\title{
Implementation of Hierarchical and Distributed Control for Discrete Event Robotic Manufacturing Systems
}

\author{
Gen'ichi Yasuda \\ Nagasaki Institute of Applied Science, Nagasaki, Japan. \\ Email: YASUDA_Genichi@NiAS.ac.jp \\ Received January $6^{\text {th }}, 2010$; revised January $22^{\text {nd }}, 2010$; accepted January $25^{\text {th }}, 2010$.
}

\begin{abstract}
The large scale and complex manufacturing systems have a hierarchical structure where a system is composed several lines with some stations and each station also have several machines and so on. In such a hierarchical structure, the controllers are geographically distributed according to their physical structure. So it is desirable to realize the hierarchical and distributed control. In this paper, a methodology is presented using Petri nets for hierarchical and distributed control. The Petri net representation of discrete event manufacturing processes is decomposed and distributed into the machine controllers, which are coordinated through communication between the coordinator and machine controllers so that the decomposed transitions fire at the same time. Implementation of a hierarchical and distributed control system is described for an example robotic manufacturing system. The demonstrations show that the proposed system can be used as an effective tool for consistent modeling and control of large and complex manufacturing systems.
\end{abstract}

Keywords: Implementation, Robotic Manufacturing Systems, Hierarchical and Distributed Control, Discrete Event Systems, Petri Nets

\section{Introduction}

Because of robot's flexibility, industrial robots have been introduced into industry to automate various operations without significant redesign. This flexibility is derived from the generality of the robot's physical structure, control and reprogrammability, but it can only be exploited if the robot can be programmed easily. In some cases, the lack of adequate programming tools makes some tasks impossible to be performed. In other cases, the cost of programming may be a significant fraction of the total cost of an application. Further, it is quite obvious that a single robot cannot perform effective tasks in an industrial environment, unless it is provided with some additional equipment. It is usually required to integrate the robot into the manufacturing system, which includes $\mathrm{NC}$ machine tools, belt conveyors, and other special purpose machines. Further, the robot often must interact with such machines, other robots or operators.

These external processes are executing in parallel and asynchronously. It is not possible to predict exactly when events of interest to the robot program may occur. The signal lines are supported by most robot systems to coor- dinate multiple robots and machines, but this is a very limited form of communication between processes. Sophisticated tasks require efficient means for coordination and for sharing the state of the system between processes. The programming system should provide a mechanism for specifying the behavior of systems more complex than a single robot. Existing robot programming systems are based on the view of a robot system as a single robot weakly linked to other machines. Many machines may be cooperating during a task. The interactions between them may be highly dynamic. No existing robot programming system adequately deals with all of these interactions. No existing computer language is adequate to deal with this kind of parallelism and real-time constraints.

The overall structure of the working area in a large and complex manufacturing system consists of one or more lines, each line consists of one or more stations, and each station (shop or cell) consists of one or more machines such as robots and intelligent machine tools. Inside of a cell, machines execute cooperation tasks such as machining, assembling and storing. Inside of a shop, cells cooperate mutually and execute more complicated tasks. Furthermore each machine consists of several motion elements. 
A task executed by a robot or an intelligent machine tool can be seen as some connection of more detailed subtasks. For example, transferring an object from a start position to a goal position is a sequence of the following subtasks; moving the hand to the start position, grasping the object, moving to the goal position, and putting it on the specified place. Thus the manufacturing system handles complicated tasks by dividing a task hierarchically in this structure, which is expected to be effective in managing cooperation tasks executed by great many machines or robots.

One of the effective methods to describe and control such systems is the Petri net which is a modeling tool for asynchronous and concurrent discrete event systems [1]. Conventional Petri net based control systems were implemented based on an overall system model. The description capability of the Petri net is very high; nevertheless, in case of manufacturing systems, the network model becomes complicated and it lacks for the readability and comprehensibility. Since in the large and complex systems, the controllers are geographically distributed according to their physical (hardware) structure, it is desirable to realize the hierarchical and distributed control.

The hierarchical and distributed control for large and complex discrete event manufacturing systems has not been implemented so far [2-4]. A Petri net model includes control algorithms, and is used to control the manufacturing process by coincidence of the behavior of the real system with the Petri net model. Thus, if it can be realized by Petri nets, the modeling, simulation and control of large and complex discrete event manufacturing systems can be consistently realized by Petri nets [5-6]. In this paper, the author presents a methodology by extended Petri nets for hierarchical and distributed control of large and complex robotic manufacturing systems, to construct the control system where the cooperation of each controller is implemented so that the aggregated behavior of the distributed system is the same as that of the original system and the task specification is completely satisfied.

\section{Discrete Event Modeling of Robotic Manufacturing Systems using Petri Nets}

A manufacturing process is characterized by the flow of workpieces or parts, which pass in ordered form through subsystems and receive appropriate operations. Each subsystem executes manufacturing operations, that is, physical transformations such as machining, assembling, or transfer operations such as loading and unloading. From the viewpoint of discrete event process control, an overall manufacturing process can be decomposed into a set of distinct activities (or events) and conditions mutually interrelated in a complex form. An activity is a single operation of a manufacturing process executed by a subsystem. A condition is a state in the process such as machine operation mode.
For example, a simple robot operation example, where the robot waits until a workpiece appears and then handles the workpiece and sends it out for the next operation, can be modeled as follows:

condition the robot is waiting

event a workpiece arrives

condition the workpiece has arrived and is waiting

event the robot starts the handling

condition the robot is handling the workpiece

event the robot finishes the handling

condition the handling has been completed

event the workpiece is sent for other operation

The above example illustrates a system where events and conditions are mutually connected. These systems are known as event-condition systems. Events simultaneously represent the end of the preceding condition and the beginning of the succeeding condition. Event-condition systems exhibit the following features:

1) Asynchronism

The system is essentially asynchronous. Events always occur when their conditions are satisfied.

\section{2) Ordering}

Before and after one condition there are always events, and each event is defined by preconditions and post conditions.

3) Parallelism

In one system two or more conditions can be held simultaneously and for this, events that do not interact may occur independently.

\section{4) Conflict}

One condition can be a precondition of various events and depending on which event is occurring, different conditions hold.

Because of these features, the following phenomena can occur in the event-condition system:

1) Deadlock occurs when the system enters into a state that is not possible for any event to occur.

2) Bumping occurs when despite the holding of a condition, the preceding event occurs. This can result in the multiple holding of that condition. When the system is free of this phenomenon, the system is called safe.

To represent discrete event manufacturing systems a modeling technique was derived from Petri nets [7-8]. Considering not only the modeling of the systems but also the actual manufacturing system control, the guarantee of safeness and the additional capability of input/output signals from/to the machines are required. The extended Petri net consists of the following six elements: 1) Place; 2) Transition; 3) Directed arc; 4) Token; 5) Gate arc; 6) Output signal arc.

A place represents a condition of a system element or action. A transition represents an event of the system. A directed arc connects a place to a transition, and its direction shows the input and output relation between them. Places and transitions are alternately connected using 
directed arcs. The number of directed arcs connected with places or transitions is not restricted. A token is placed in a place to indicate that the condition corresponding to the place is holding.

A gate arc connects a transition with a signal source, and depending on the signal, it either permits or inhibits the occurrence of the event which corresponds to the connected transition. Gate arcs are classified as permissive or inhibitive, and internal or external. An output signal arc sends the signal from a place to an external machine. A transition is enabled if and only if it satisfies all the following conditions:

1) It does not have any output place filled with a token.

2) It does not have any empty input place.

3) It does not have any internal permissive arc signaling 0 .

4) It does not have any internal inhibitive arc signaling 1 .

An enabled transition may fire when it does not have any external permissive arc signaling 0 nor any external inhibitive arc signaling 1 . The firing of a transition removes tokens from all its input places and put a token in each output place connected to it. The assignment of tokens into the places of a Petri net is called marking and it represents the system state. In any initial marking, there must not exist more than one token in a place. According to these rules, the number of tokens in a place never exceeds one, thus, the Petri net is essentially a safe graph.

If a place has two or more input transitions or output transitions, these transitions may be in conflict for firing. When two or more transitions are enabled only one transition should fire using some arbitration rule. By the representation of the activity contents and control strategies in detail, features of discrete event manufacturing systems such as ordering, parallelism, asynchronism, concurrency and conflict can be concretely described through the extended Petri net.

\section{Design of Hierarchical and Distributed Control}

The overall procedure for the design and implementation of hierarchical and distributed control is summarized as shown in Figure 1. The basic procedures of modeling and decomposition of robotic manufacturing systems are shown. A global, conceptual Petri net model is first chosen which describes the aggregate manufacturing process. At the conceptual level each task specification is represented as a place of the Petri net as shown, where the activity of each equipment is also represented as a place.

Based on the hierarchical approach, the Petri net is translated into detailed subnets by stepwise refinements from the highest system control level to the lowest machine control level. At each step of detailed specification, some parts of the Petri net, transitions or places, are substituted by a subnet in a manner, which maintains the structural properties.

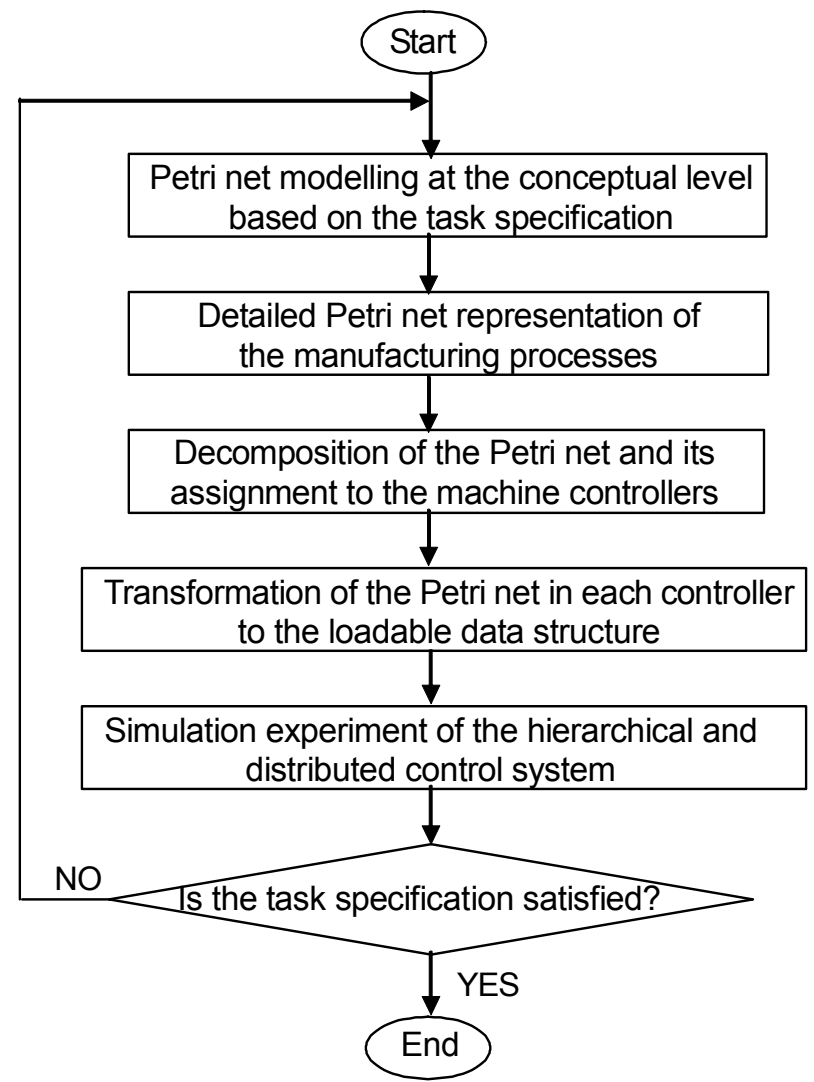

Figure 1. Flow chart of Petri net based implementation of hierarchical and distributed control system

It is natural to implement a hierarchical and distributed control system, where one controller is allocated to each control layer or block. For the manufacturing system, an example structure of hierarchical and distributed control is composed of one station controller and three machine controllers as shown in Figure 2, although each robot may be controlled by one robot controller. The detailed Petri net is decomposed into subnets, which are executed by each machine controller.

In the decomposition procedure, a transition may be divided and distributed into different machine controllers as shown in Figure 3. The machine controllers should be coordinated so that these transitions fire in union. Decomposed transitions are called global transitions, and other transitions are called local transitions.

Decomposed transitions must function in union, that is, the aggregate behavior of decomposed subnets should be the same as that of the original Petri net. By the Petri net model, the state of the discrete event system is represented as the marking of tokens, and firing of any transition brings about change to the next state. So the firing condition and state (marking) change before decomposition should be the same as those after decomposition. The firability condition and external gate condition of a transition $j$ before decomposition are described as follows: 


$$
\begin{array}{r}
t_{j}(k)=\bigcap_{m=1}^{M} p_{j, m}^{I}(k) \wedge \bigcap_{n=1}^{N} \overline{p_{j, n}^{O}(k)} \wedge \\
\bigcap_{q=1}^{Q} g_{j, q}^{I P}(k) \wedge \bigcap_{r=1}^{R} \overline{g_{j, r}^{I I}(k)} \\
g_{j}^{E}(k)=\bigcap_{u=1}^{U} g_{j, u}^{E P}(k) \wedge \bigcap_{v=1}^{V} \overline{g_{j, v}^{E I}(k)}
\end{array}
$$

where,

$M$ : input place set of transition $j$;

$p_{j, m}^{I}(k):$ state of input place $m$ of transition $j$ at time sequence $k$;

$N$ : output place set of transition $j$;

$p_{j, n}^{O}(k):$ state of output place $n$ of transition $j$ at time sequence $k$;

$Q$ : internal permissive gate signal set of transition $j$;

$g_{j, q}^{I P}(k):$ internal permissive gate signal variable $q$ of transition $j$ at time sequence $k$;

$R$ : internal inhibitive gate signal set of transition $j$;

$g_{j, r}^{I I}(k)$ : internal inhibitive gate signal variable $r$ of transition $j$ at time sequence $k$;

$U$ : external permissive gate signal set of transition $j$;

$g_{j, u}^{E P}(k)$ : external permissive gate signal variable $u$ of transition $j$ at time sequence $k$;

$V$ : external inhibitive gate signal set of transition $j$;

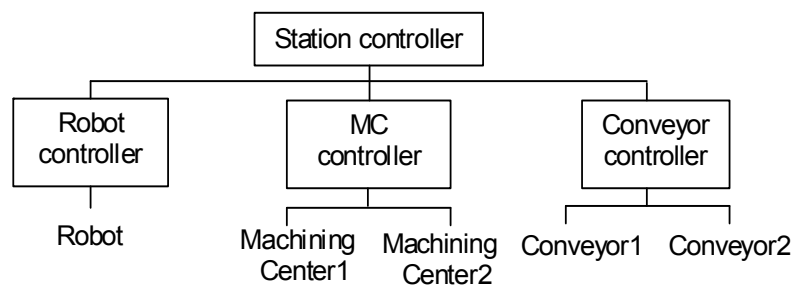

Figure 2. Example structure of distributed control system
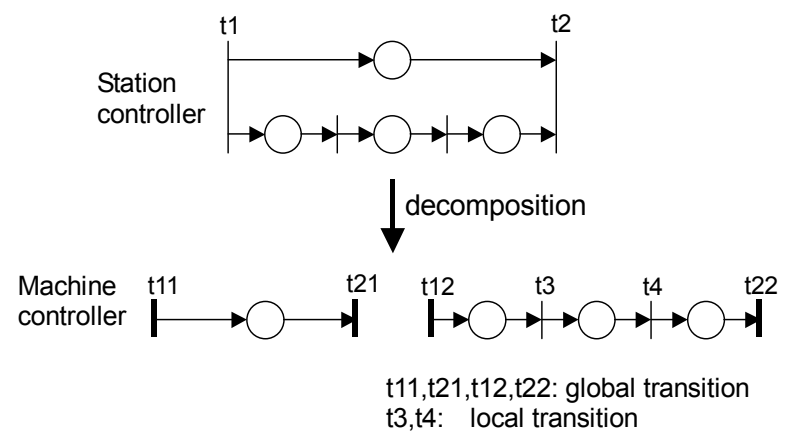

Figure 3. Decomposition of transition $g_{j, v}^{E I}(k)$ : external inhibitive gate signal variable $v$ of transition $j$ at time sequence $k$;

The state (marking) change, that is, the addition or removal of a token of a place, is described as follows:

$$
\begin{gathered}
p_{j, m}^{I}(k+1)=p_{j, m}^{I}(k) \wedge \overline{\left(t_{j}(k) \wedge g_{j}^{E}(k)\right)} \\
p_{j, n}^{O}(k+1)=p_{j, n}^{O}(k) \vee\left(t_{j}(k) \wedge g_{j}^{E}(k)\right)
\end{gathered}
$$

If transition $j$ is divided into $s$ transitions $j 1, j 2,,, j \mathrm{~s}$, the firability condition of a transition after decomposition is described as follows:

$$
\begin{array}{r}
t_{j s u b}(k)=\bigcap_{m=1}^{M s u b} p_{j s u b, m}^{I}(k) \wedge \bigcap_{n=1}^{N s u b} \overline{p_{j s u b, n}^{O}(k) \wedge} \\
\bigcap_{q=1}^{Q s u b} g_{j s u b, q}^{I P}(k) \wedge \bigcap_{r=1}^{R s u b} \overline{g_{j s u b, r}^{I I}(k)} \\
g_{j s u b}^{E}(k)=\bigcap_{u=1}^{U s u b} g_{j s u b, u}^{E P}(k) \wedge \bigcap_{v=1}^{V s u b} \overline{g_{j s u b, v}^{E I}(k)}
\end{array}
$$

From Equation (1) and Equation (5),

$$
t_{j}(k)=\bigcap_{s u b=1}^{S} t_{j s u b}(k)
$$

From Equation (2) and Equation (6),

$$
g_{j}^{E}(k)=\bigcap_{s u b=1}^{S} g_{j s u b}^{E}(k)
$$

where,

$S$ : total number of subnets

$M s u b$ : input place set of transition jsub of subnet sub ;

$p_{j s u b, m}^{I}(k)$ : state of input place $m$ of transition $j s u b$ of subnet $s u b$ at time sequence $k$;

$N s u b$ : output place set of transition jsub of subnet sub;

$p_{j s u b, n}^{O}(k):$ state of output place $n$ of transition $j s u b$ of subnet $s u b$ at time sequence $k$;

Qsub: internal permissive gate signal set of transition jsub of subnet $s u b$;

Rsub: internal inhibitive gate signal set of transition jsub of subnet $s u b$;

Usub: external permissive gate signal set of transition jsub of subnet $s u b$;

$V s u b$ : external permissive gate signal set of transition jsub of subnet $s u b$; 
The addition or removal of a token of a place connected to a decomposed transition is described as follows:

$$
\begin{aligned}
& p_{j s u b, m}^{I}(k+1)=p_{j s u b, m}^{I}(k) \wedge \overline{\left(t_{j}(k) \wedge g_{j}^{E}(k)\right)} \\
& p_{j s u b, n}^{O}(k+1)=p_{j s u b, n}^{O}(k) \vee\left(t_{j}(k) \wedge g_{j}^{E}(k)\right)
\end{aligned}
$$

Consequently it is proved that the firability condition of the original transition is equal to AND operation of firability conditions of decomposed transitions. If and only if all of the decomposed transitions are enabled, then the global transitions are enabled. To exploit the above results, the coordinator program has been introduced to coordinate the decomposed subnets so that the aggregate behavior of decomposed subnets is the same as that of the original Petri net.

There may exist a place which has plural input transitions and/or plural output transitions. This place is called a conflict place. The transitions connected to a conflict place are in conflict when some of them are enabled at the same time. In this case, only one of them fires and the others become disabled. The choice for firing is done arbitrarily using an arbiter program.

In case that a transition in conflict with other transitions is decomposed as shown in Figure 4, these transitions should be coordinated by the station controller. If arbitration of the transitions is performed independently in separate subnets, the results may be inconsistent with the original rule of arbitration. Therefore the transitions should be arbitrated together as a group. On the other hand, arbitration of local transitions in conflict is performed by local machine controllers.

The Petri net based control structure with introduction of coordinator is shown in Figure 5. The control software is distributed into the station controller and machine controllers. The station controller is composed of the Petri net based controller and the coordinator. The conceptual Petri net model is allocated to the Petri net based controller for management of the overall system. For cooperative or exclusive tasks between robots, global transitions at the station controller are used to communicate the status of the robots. The detailed Petri net models are allocated to the Petri net based controllers in the machine controllers. Each machine controller directly monitors and controls the sensors and actuators of its machine.

The control of the overall system is achieved by coordinating these Petri net based controllers. System coordination is performed through communication between the coordinator in the station controller and the Petri net based controllers in the machine controllers as the following steps.

1) When each machine controller receives the start signal from the coordinator, it tests the firability of all

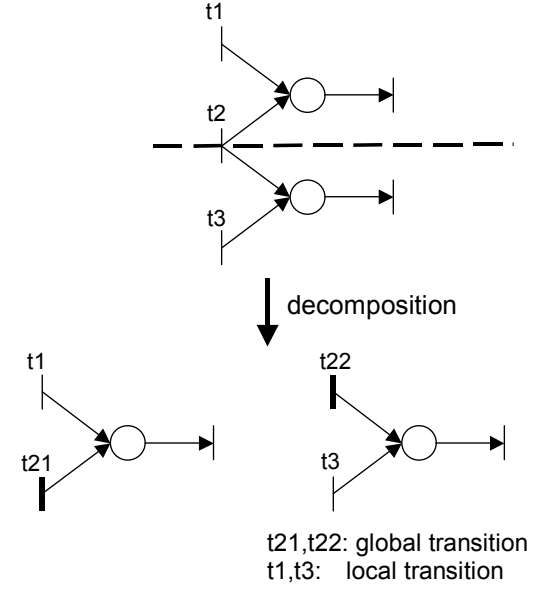

Figure 4. Decomposition of transition in conflict

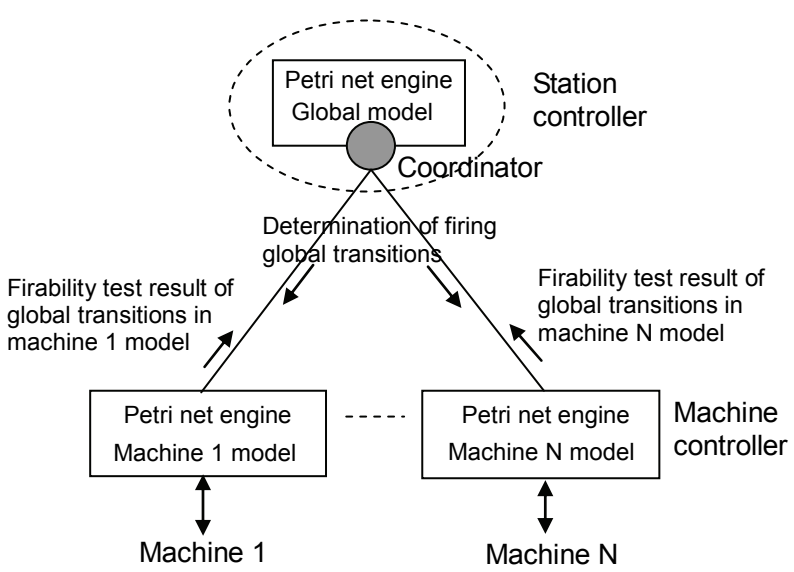

Figure 5. Petri net based control structure with coordinator

transitions in its own Petri net, and sends the information on the global transitions and the end signal to the coordinator.

2) The coordinator tests the firability of the global transitions, arbitrates conflicts among global and local transitions, and sends the names of firing global transitions and the end signal to the machine controllers.

3) Each machine controller arbitrates conflicts among local transitions using the information from the coordinator, generates a new marking, and sends the end signal to the coordinator.

4) When the coordinator receives the end signal from all the machine controllers, it sends the output command to the machine controllers.

5) Each machine controller outputs the control signals to its actuators.

Multilevel hierarchical and distributed control for large and complex manufacturing systems can be constructed such that the control system structure corresponds to the hierarchical and distributed structure of the general manufacturing system. The coordination mecha- 
nism is implemented in each layer repeatedly as shown in Figure 6. The overall system is consistently controlled, such that a coordinator in a layer coordinates one-level lower Petri net based controllers and is coordinated by the one-level upper coordinator.

The details of coordination in a two-level control system composed of a global controller and several local controllers have been implemented as shown in Figure 7.

\section{Implementation of Control System}

\subsection{Example of Workstation Task}

The basic procedures of modeling and decomposition of robotic manufacturing systems are shown through a simple example. The example robotic manufacturing system is composed of two input conveyors, two machining centers, one handling robot, and one output conveyor as shown in Figure 8.

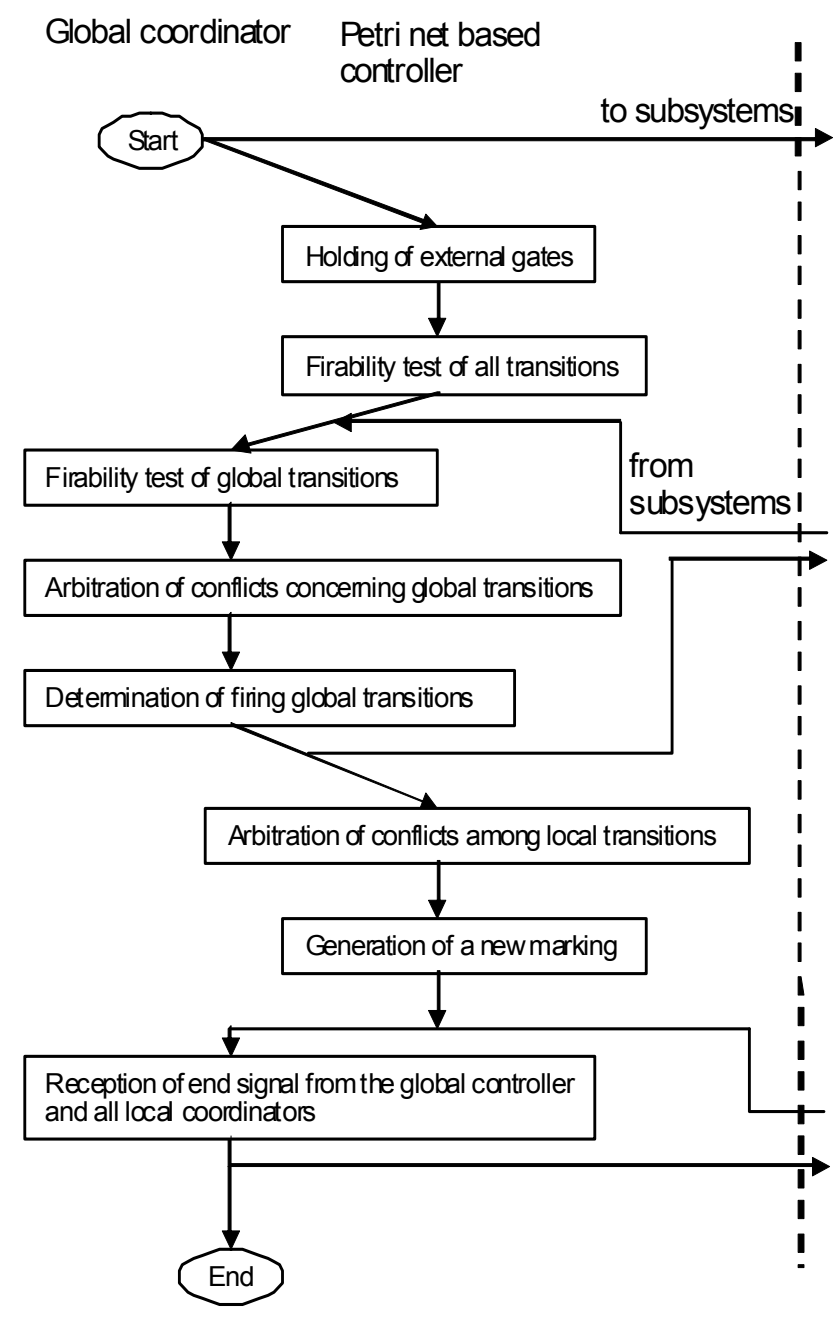

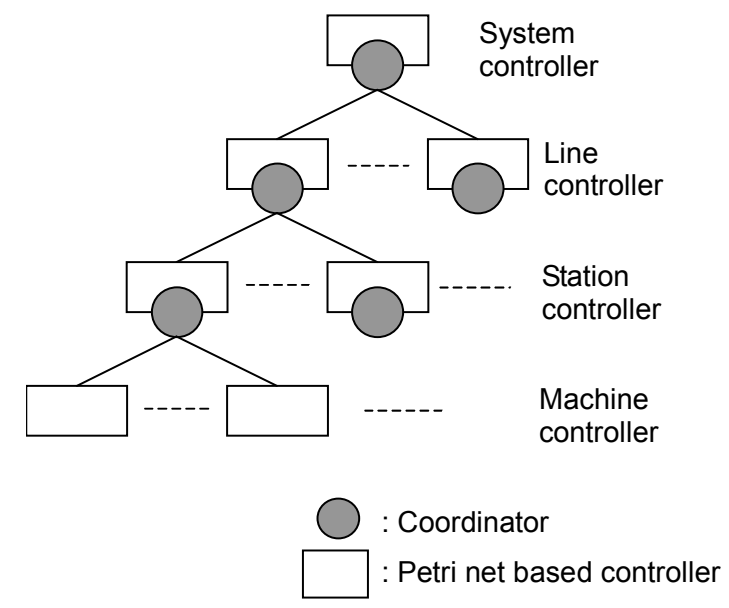

Figure 6. Hierarchical and distributed control structure for overall manufacturing system

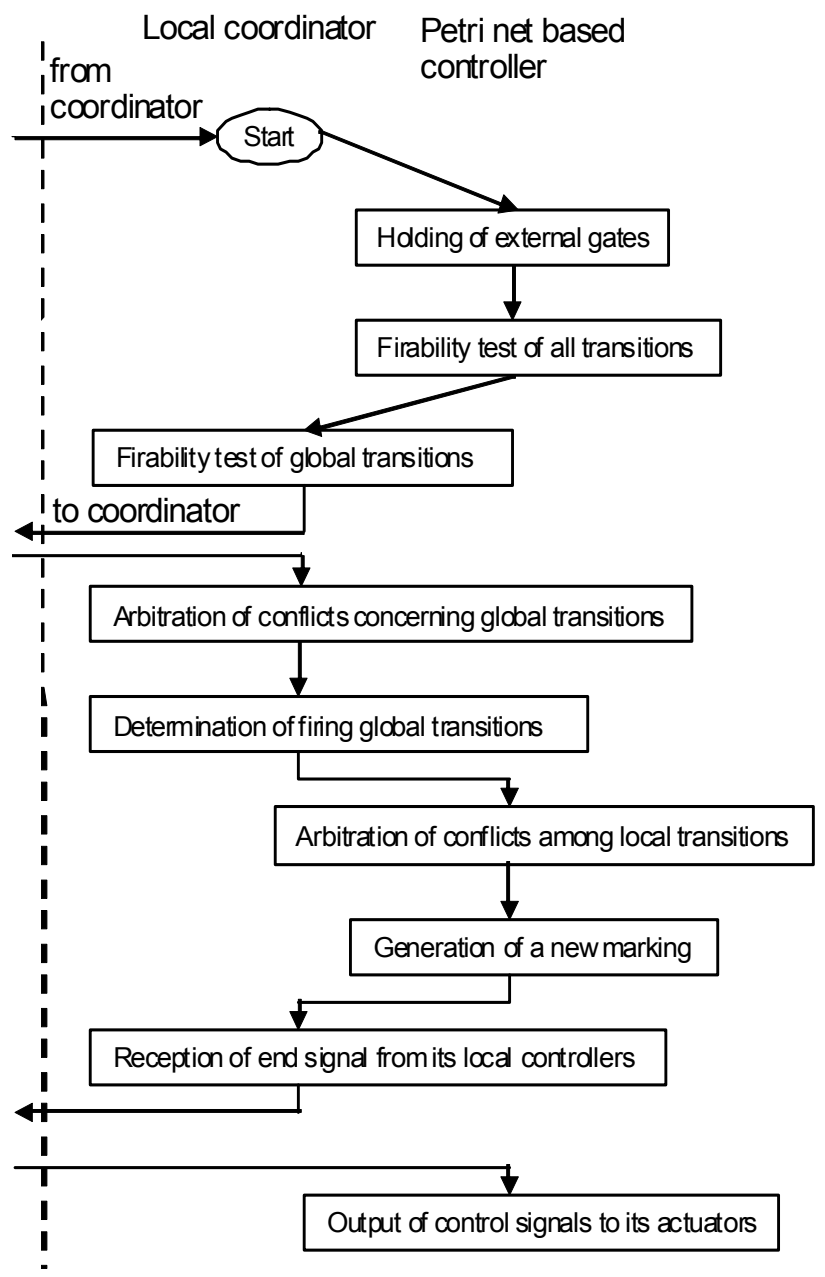

Figure 7. Flowchart of coordination in two-level control system 


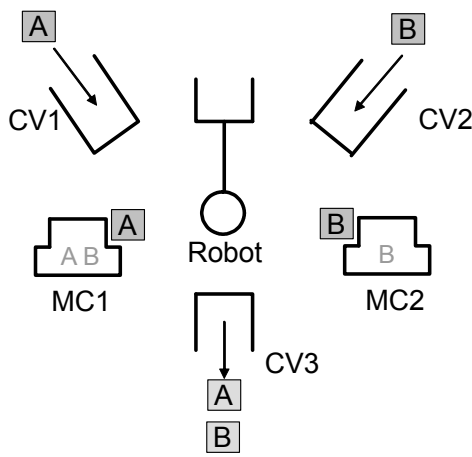

Figure 8. Example of robotic manufacturing system

The task specification of each equipment with the names of subtasks is as follows.

1) The conveyor CV1 carries a workpiece of type A into the workcell (CIN_A).

2) The conveyor $\mathrm{C} \overline{\mathrm{V}} 2$ carries a workpiece of type $B$ into the workcell (CIN_B).

3) The robot loads the workpiece of type A into the machining center MC1 (LDA_MC1).

4) The robot loads the workpiece of type $B$ into the machining center MC1 (LDB MC1).

5) The robot loads the workpiece of type $B$ into the machining center MC2 (LDB_MC2).

6) The machining centers process the workpieces each (PR_MC1, PR_MC2).

7) The robot unloads the processed workpiece from the machining center $\mathrm{MC1}$ and carries them to the conveyor CV3 (UNLD_MC1).

8) The robot unloads the processed workpiece from the machining center MC2 and carries them to the conveyor CV3 (UNLD_MC2).

9) The conveyor CV3 carries the workpiece away (COUT).

\subsection{Petri Net Based Modeling}

From the viewpoint of the flow of workpieces, the task specification is summarized by Petri nets as shown in Figure 9.

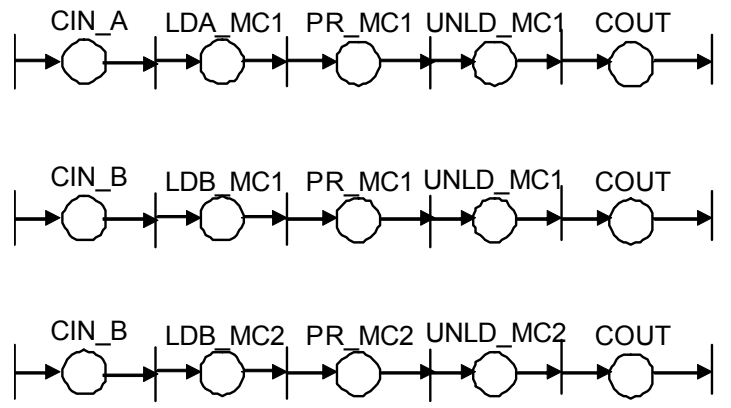

Figure 9. Petri net representation of the task specification of the example system
A global, conceptual Petri net model is first defined which describes the aggregate manufacturing process. At the conceptual level each task specification is represented as a place of the Petri net as shown in Figure 10, where the activity of each equipment is also represented as a place. Activities of the conveyor CV2, the machining center $\mathrm{MC} 1$ and the robot should be arbitrated based on the global Petri net model, because the places have two or more input/output transitions.

Based on the hierarchical approach, Petri nets are translated into detailed subnets by stepwise refinements from the highest system control level to the lowest machine control level [6]. At each step of detailed specification, some places of the Petri net are substituted by a subnet in a manner, which maintains the structural properties. Figure 11 shows the detailed Petri net representation of subtasks: loading, processing and unloading in Figure 10.

The transitions among associated machines in the detailed Petri net representations imply the cooperative control structure in the overall system. For example, loading a workpiece of type A necessitates the cooperative or synchronized activities among the conveyor CV1, the machining center $\mathrm{MC1}$, and the robot. First, "forward" operation to carry a workpiece is performed by the
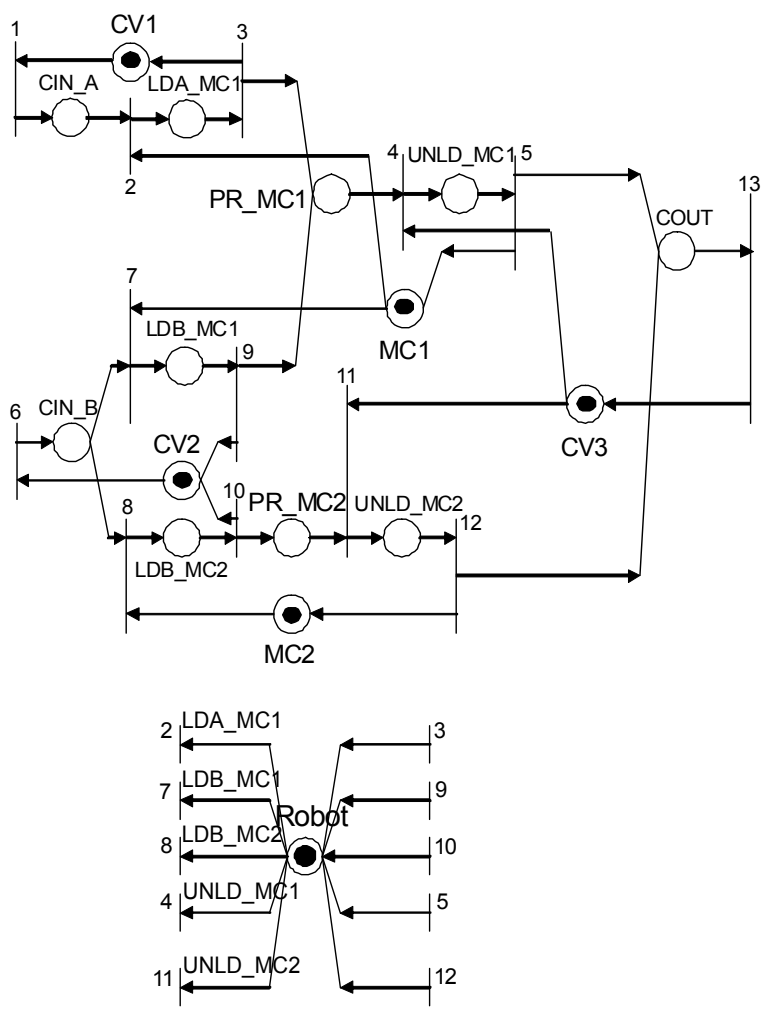

Figure 10. Petri net representation of the example system at the conceptual level 


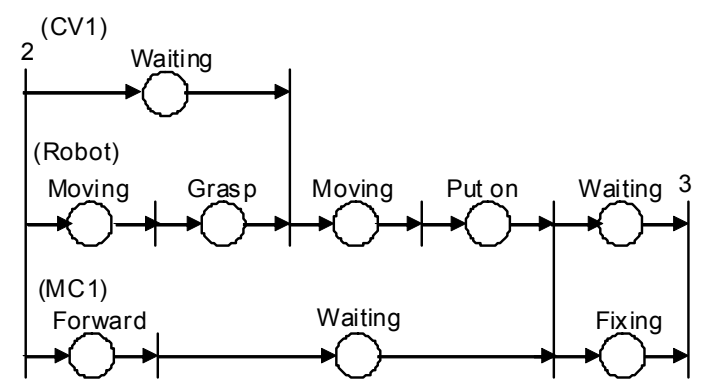

(a) Loading A to MC1 (LDA_MC1)

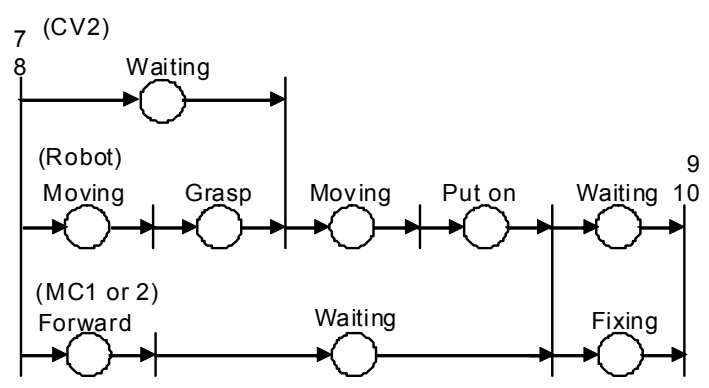

(b).Loading B into MC1 or MC2 (LDB_MC1, LDB_MC2)

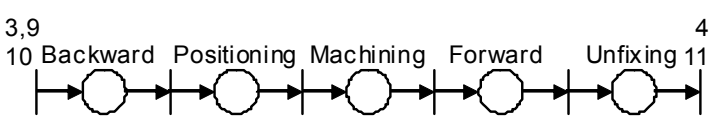

(c) Processing (PR_MC1, PR_MC2)

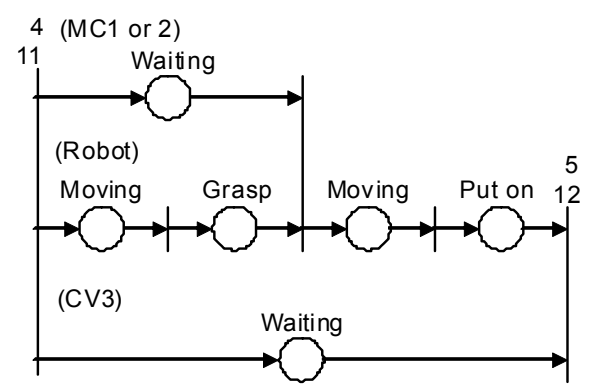

(d) Unloading A or B (UNLD_MC1, UNLD_MC2)

Figure 11. Detailed Petri net representation of subtasks

conveyor CV1. At the end of "forward" operation, when the robot is free, the "loading" operation is started. The conveyor CV1 starts waiting, the robot starts moving to grasp the workpiece, and the machining center starts moving forward to get the workpiece from the robot. After holding the workpiece, the robot starts moving to put it on the machining center and the conveyor CV1 is free. After putting on, the machining center starts fixing the workpiece, while the robot is waiting. After fixing the workpiece, the "loading" operation is finished.

\subsection{Control System Design and Experiments}

For the manufacturing system, an example structure of hierarchical and distributed control is composed of one station controller and three machine controllers (Figure 2). The Petri net executed in each machine controller is shown in Figure 12, simply by extracting the specified sequences of subtasks in the detailed Petri nets. For the implementation of the Petri net based control algorithm,

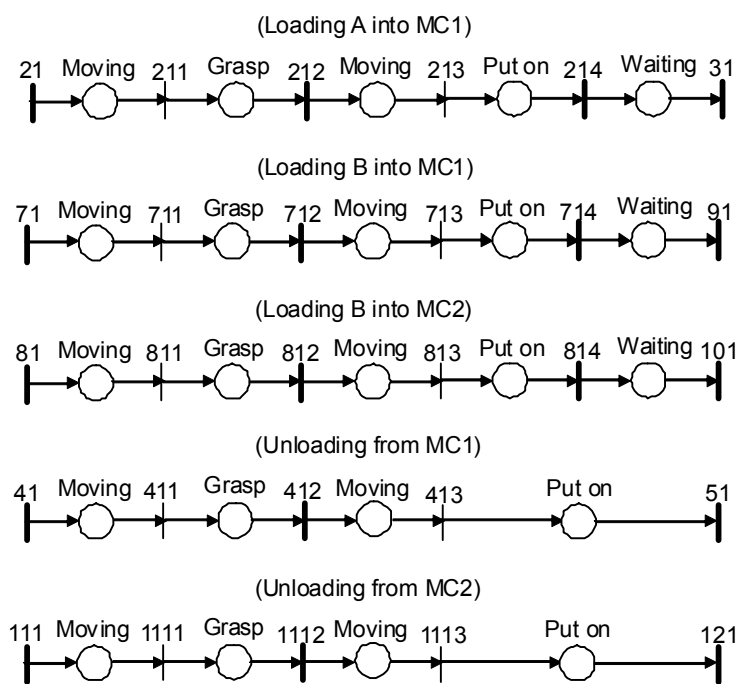

(a) Robot controller

(MC1: Fixing and processing A or B)

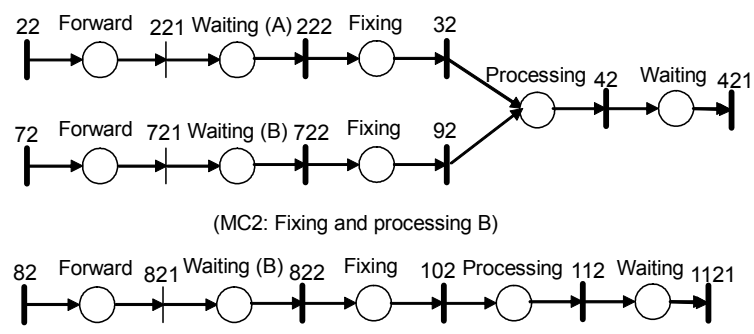

(b) MC controller

(CV1: Carrying A to workcell; MC1)

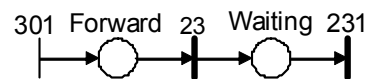

(CV2: Carrying B to workcell; MC1 or MC2)

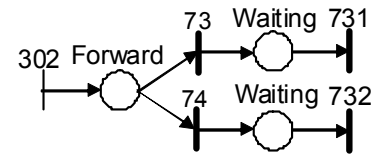

(CV3: Carrying out from MC1 or MC2)

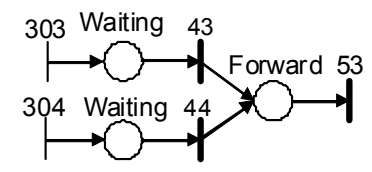

(c) Conveyor controller

Figure 12. Petri net representation of machine controllers ( \: global transition) 
a transition of a Petri net model is defined using the names of its input places and output places; for example, $\mathrm{t} 1-1=\mathrm{p} 1-1,-\mathrm{p} 1-11$, where the transition no.1 (t1-1) of the subsystem no. 1 is connected to the input place no. 1 and the output place no.11. Using the names of transitions, global transitions are defined; for example, $\mathrm{G} 1=\mathrm{t} 1-1$, $\mathrm{t} 2-1$, t3-1 indicates that the global transition G1 is composed of the transition no.1 of the subsystem no.1 (Robot controller), the transition no.1 of the subsystem no.2 (MC controller), and the transition no.1 of the subsystem no. 3 (Conveyor controller). Then, the global transitions with comments of the example control system are as follows.

$\mathrm{G} 1=\mathrm{t} 1-21, \mathrm{t} 2-22, \mathrm{t} 3-23$ (start of loading A from CV1);

$\mathrm{G} 2=\mathrm{t} 1-212, \mathrm{t} 3-231 \quad$ (end of grasp A on CV1);

$\mathrm{G} 3=\mathrm{t} 1-214, \mathrm{t} 2-222 \quad$ (end of put A on MC1);

$\mathrm{G} 4=\mathrm{t} 1-31, \mathrm{t} 2-32 \quad$ (end of loading $\mathrm{A}$ into $\mathrm{MC} 1$ );

$\mathrm{G} 5=\mathrm{t} 1-71, \mathrm{t} 2-72, \mathrm{t} 3-73$ (start of loading B from CV2);

$\mathrm{G} 6=\mathrm{t} 1-712, \mathrm{t} 3-731 \quad$ (end of grasp B on CV2);

$\mathrm{G} 7=\mathrm{t} 1-714, \mathrm{t} 2-722 \quad$ (end of put $\mathrm{B}$ on $\mathrm{MC} 1$ );

$\mathrm{G} 8=\mathrm{t} 1-91, \mathrm{t} 2-92 \quad$ (end of loading $\mathrm{B}$ into $\mathrm{MC1}$ );

$\mathrm{G} 9=\mathrm{t} 1-81, \mathrm{t} 2-82, \mathrm{t} 3-74$ (start of loading B from CV2);

$\mathrm{G} 10=\mathrm{t} 1-812, \mathrm{t} 3-731 \quad$ (end of grasp B on CV2);

$\mathrm{G} 11=\mathrm{t} 1-814, \mathrm{t} 2-822 \quad$ (end of put $\mathrm{B}$ on $\mathrm{MC} 2$ );

$\mathrm{G} 12=\mathrm{t} 1-101, \mathrm{t} 2-102 \quad$ (end of loading B into MC2);

$\mathrm{G} 13=\mathrm{t} 1-41, \mathrm{t} 2-42, \mathrm{t} 3-303$ (start of unloading from MC1);

$\mathrm{G} 14=\mathrm{t} 1-412$, $\mathrm{t} 2-421 \quad$ (end of grasp on MC1);

$\mathrm{G} 15=\mathrm{t} 1-51, \mathrm{t} 3-43 \quad$ (end of put on CV3);

$\mathrm{G} 16=\mathrm{t} 1-111, \mathrm{t} 2-112, \mathrm{t} 3-304$ (start of unloading from $\mathrm{MC} 2$ );

$\mathrm{G} 17=\mathrm{t} 1-1112, \mathrm{t} 2-1121$ (end of grasp on MC2);

$\mathrm{G} 18=\mathrm{t} 1-121, \mathrm{t} 3-44 \quad($ end of put on CV3);
The hierarchical and distributed control system has been realized using a set of PCs. Each machine controller is implemented on a dedicated PC. The station controller is implemented on another PC. Communications among the controllers are performed using serial communication interfaces. A Petri net model includes control algorithms, and is used to control the manufacturing process by coincidence of the behavior of the real system with the Petri net model.

The names of global transitions and their conflict relations are loaded into the coordinator in the station controller. The connection structure of a decomposed Petri net model and conflict relations among local transitions are loaded into the Petri net based controller in a machine controller. By executing the coordinator and Petri net based controllers algorithms based on loaded information, simulation experiments have been performed. The Petri net simulators in the machine controllers initiate the execution of the subtasks attached to the fired transitions through the serial interface to the robot or other external machine. When a task ends its activity, it informs the simulator to proceed with the next activations by the external permissive gate arc. A machine controller controls one or more machines or robots using multithreaded programming [9]. Experimental results show that the decomposed transitions fire at the same time as the original transition of the detailed Petri net of the whole system task. Firing transitions and marking of tokens can be directly observed on the display at each time sequence using the simulator as shown in Figure 13 [10].

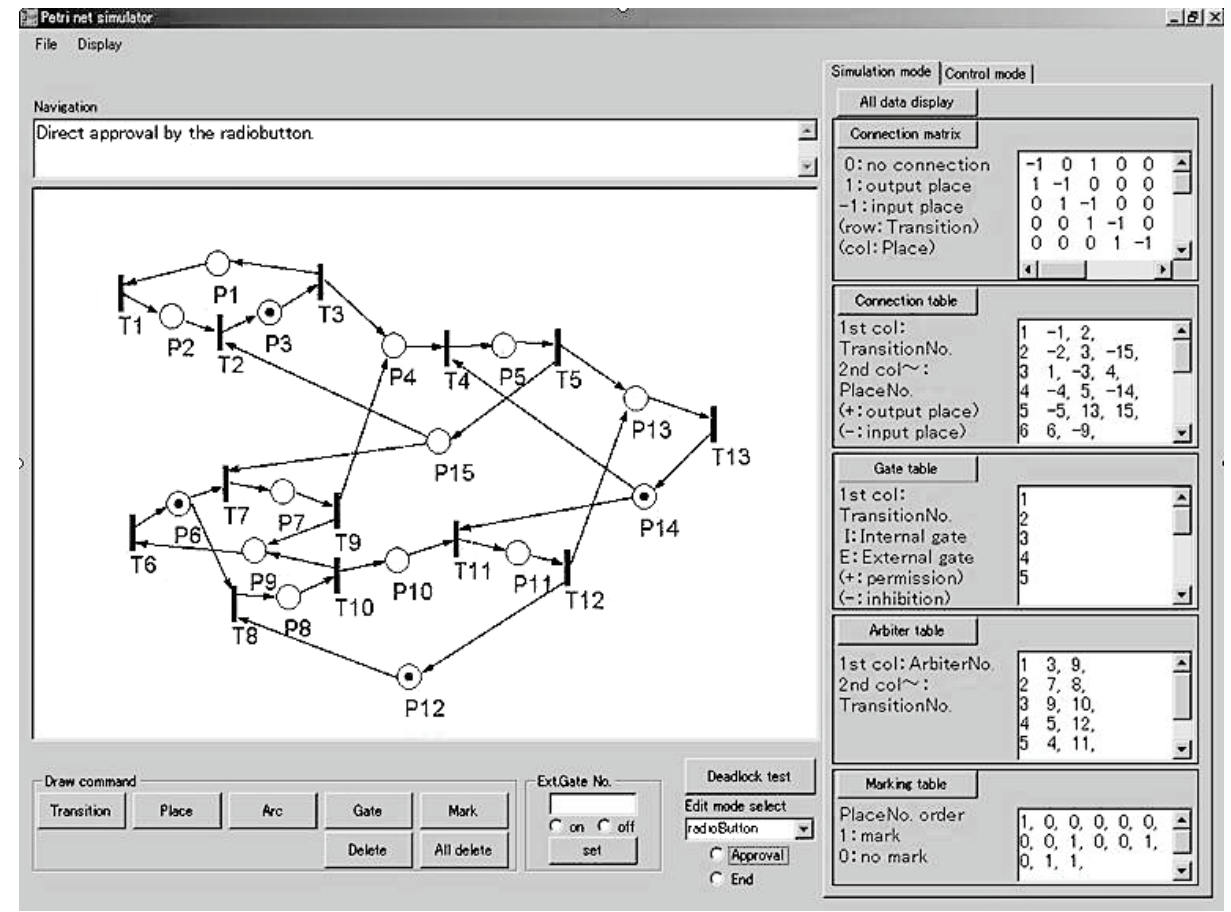

Figure 13. View of Petri net simulator at the station controller 


\section{Conclusions}

A methodology to construct hierarchical and distributed control systems, which correspond to the structure of manufacturing systems, has been presented. The overall control structure of an example robotic manufacturing system was implemented using a communication network of PCs, where each machine controller is realized on a dedicated PC. The Petri net based control software is distributed into the station controller and machine controllers; the station controller executes the conceptual Petri net, and the machine controllers execute decomposed subnets. The controllers are arranged according to the hierarchical and distributed nature of the manufacturing system. The control software does not use the overall system model, and the decomposed Petri net model in each machine controller is not so large and easily manageable. Machine controllers are coordinated such that decomposed transitions fire at the same time and the task specification is completely satisfied. The Petri net model includes the control algorithm; control is executed in order that the behaviour of the Petri net model is in correspondence with that of the real system. Thus modeling, simulation and control of large and complex manufacturing systems can be performed consistently using Petri nets. The experimental control system uses conventional PCs with serial interfaces, but the performance of the control system can be improved using dual port shared memory and high-speed serial interfaces for communication between controllers.

\section{REFERENCES}

[1] W. Reisig, "Petri Nets," Springer-Verlag, Berlin, 1985.
[2] M. Silva, "Petri Nets and Flexible Manufacturing," In G. Rozenberg, Ed., Advances in Petri Nets 1989, Lecture Notes in Computer Science, Springer-Verlag, Vol. 424, 1990, pp. 374-417.

[3] A. D. Desrochers and R. Y. Al-Jaar, "Applications of Petri Nets in Manufacturing Systems: Modeling, Control and Performance Analysis," IEEE Press, 1995.

[4] E. J. Lee, A. Togueni, and N. Dangoumau, "A Petri Net Based Decentralized Synthesis Approach for the Control of Flexible Manufacturing Systems," Proceedings of the IMACS Multiconference Computational Engineering in Systems Applications, Lille, 2006.

[5] G. Bruno, "Model-based Software Engineering," Chapman \& Hall, 1995.

[6] V. O. Pinci and R. M. Shapiro, "An Integrated Software Development Methodology Based on Hierarchical Colored Petri Nets," In G. Rozenberg, Ed., Advances in Petri Nets 1991, Lecture Notes in Computer Science, Springer Verlag, Vol. 524, 1991, pp. 227-252.

[7] K. Hasegawa, K. Takahashi and P. E. Miyagi, "Application of the Mark Flow Graph to Represent Discrete Event Production Systems and System Control," Transactions of the SICE, Vol. 24, No. 1, 1988, pp. 69-75.

[8] P. E. Miyagi, K. Hasegawa and K. Takahashi, "A Programming Language for Discrete Event Production Systems Based on Production Flow Schema and Mark Flow Graph," Transactions of the SICE, Vol. 24, No. 2, 1988, pp. 183-190.

[9] G. Yasuda, "Distributed Control of Multiple Cooperating Robot Agents Using Multithreaded Programming," Proceedings of the 16th International Conference on Production Research, Prague, 2001.

[10] G.Yasuda, "Implementation of Distributed Cooperative Control for Industrial Robot Systems Using Petri Nets," Preprints of the 9th IFAC Symposium on Robot Control, Gifu, 2009, pp. 433-438. 